\begin{tabular}{|l|l|}
\hline $\begin{array}{l}\text { Instituto de } \\
\text { Geriatria e Gerontologia }\end{array}$ & $\begin{array}{l}\text { Pan American Journal of Aging Research } \\
\text { PAJAR, Porto Alegre, v. 9, p. 1-10, jan.-dez. } 2021 \\
\text { ISSN-L: 2357-9641 }\end{array}$ \\
\hline http://dx.doi.org/10.15448/2357-9641.2021.1.40298 & \\
\cline { 1 - 2 } &
\end{tabular}

ARTIGO ORIGINAL

\title{
Prevalência e fatores associados ao uso de benzodiazepínicos em idosos atendidos em um ambulatório especializado em Porto Alegre, Brasil
}

\author{
Prevalence and factors associated with the use of benzodiazepines in elderly in a \\ specialized ambulatory in Porto Alegre, Brazil \\ Prevalencia y factores asociados con el uso de benzodiazepinas en ancianos en un \\ ambulatorio especializado en Porto Alegre, Brasil
}

\section{Isabella Serafin Couto ${ }^{1}$ \\ orcid.org/0000-0002-0382-5118 \\ isabellacouto@gmail.com}

\section{Vanessa Sgnaolin ${ }^{1}$ \\ orcid.org/0000-0002-9914-7146 vanessa.sgnaolin@pucrs.br}

Paula Engroff ${ }^{1}$

orcid.org/0000-0002-3639-545X

paula.engroff@pucrs.br

\section{Letícia Güenter \\ Dannebrock ${ }^{1}$}

orcid.org/0000-0003-1615-1893

leticiaguenter@hotmail.com

\section{Luiz Gustavo}

Guilhermano $^{1}$

orcid.org/000-0001-6664-7323

luiz.guilhermano@pucrs.br

\section{Alfredo Cataldo Neto ${ }^{1}$}

orcid.org/0000-0002-8082-1866 cataldo@pucrs.br

Recebido em: 04/03/2021 Aprovado em: 23/08/2021 Publicado em: 20/10/2021

\section{(c) (i)}

Artigo está licenciado sob forma de uma licença Creative Commons Atribuição 4.0 Internacional.
Resumo

Objetivos: verificar a prevalência de uso de BZDs na população idosa atendida em um ambulatório de psiquiatria geriátrica de um hospital universitário em Porto Alegre, Brasil.

Métodos: estudo transversal realizado no ambulatório de psiquiatria geriátrica. A coleta de dados ocorreu durante a primeira consulta dos indivíduos com idade igual ou superior a 60 anos. Os dados avaliados compreendem questões sociodemográficas, clínicas, de saúde e uso de medicamentos. Para análise estatística foi utilizado o programa SPSS (do inglês, Statistical Package for the Social Sciences). Resultados: um total de 295 idosos foram incluidos no estudo. A média de idade foi de $72,1 \pm 8,3$ anos, sendo na maioria mulheres (76,6\%), com escolaridade de 5 a 8 e 9 a 12 anos de estudo (ambos com a mesma frequência, $28,9 \%$ ), casados $(45,0 \%)$, residindo com familiar (34,4\%), aposentados $(79,8 \%)$. A prevalência de utilização de BZD foi de 33,6\% ( $n=99$ ). A queixa principal de ansiedade e o diagnóstico de transtornos ansiosos foram associadas ao uso de BZDs.

Conclusões: a prevalência do uso de BZDs foi alta e associada à queixa principal de ansiedade e aos diagnósticos de transtornos ansiosos e depressivos, mesmo esses sendo considerados medicamentos potencialmente inapropriados e com alto risco de complicações. O tratamento da população geriátrica, muitas vezes, é colocado em segundo plano, não recebendo devidamente os cuidados direcionados às demandas especificas que esses indivíduos necessitam. O conhecimento epidemiológico da população idosa expõe características e auxilia na elaboração de plano terapêutico diferenciado.

Palavras-chave: idoso, psiquiatria geriátrica, receptores benzodiazepínicos, lista de medicamentos potencialmente inapropriados.

\section{Abstract}

Aims: to verify the prevalence of BZDs use in the elderly population treated at an outpatient clinic for geriatric psychiatry at a university hospital in Porto Alegre, Brazil. Methods: cross-sectional study carried out at the geriatric psychiatric outpatient clinic. Data collection occurred during the first consultation of individuals aged 60 years or over. The evaluated data comprise sociodemographic, clinical, health and medication use issues. For statistical analysis the SPSS program (in English, Statistical Package for the Social Sciences) was used.

Results: a total of 295 elderly people were included in the study. The average age was $72.1 \pm 8.3$ years, mostly women (76.6\%), with schooling from 5 to 8 and 9 to 12 years of study (both with the same frequency, $28.9 \%$ ), married (45.0\%), living with a family member $(34.4 \%$ ), retired $(79.8 \%$ ). The prevalence of BZDs use was $33.6 \%$ (n =99). The main complaint of anxiety and the diagnosis of anxiety disorders were 
associated with the use of BZDs.

Conclusions: the prevalence of the use of BZDs was high and associated with the main complaint of anxiety and the diagnoses of anxiety and depressive disorders, even though these are considered potentially inappropriate medications and with a high risk of complications. The treatment of the geriatric population is often placed in the background, not properly receiving care directed to the specific demands that these individuals need. The epidemiological knowledge of the elderly population exposes characteristics and assists in the development of a differentiated therapeutic plan.

Keywords: aged, geriatric psychiatry, benzodiazepine receptor, potentially inappropriate medication list.

\section{Resumen}

Objetivos: verificar la prevalencia del uso de BZD en la población anciana atendida en un ambulatorio de psiquiatría geriátrica en un hospital universitario de Porto Alegre, Brasil.

Métodos: estudio transversal realizado en el ambulatorio psiquiátrico geriátrico. La recogida de datos se produjo durante la primera consulta de personas de 60 años o más. Los datos evaluados comprenden aspectos sociodemográficos, clínicos, de salud y de uso de medicamentos. Para el análisis estadístico se utilizó el programa SPSS (en inglés, Statistical Package for the Social Sciences).

Resultados: se incluyó en el estudio a un total de 295 ancianos. La edad promedio fue de 72,1 $\pm 8,3$ años, mayoritariamente mujeres $(76,6 \%)$, con escolaridad de 5 a 8 y de 9 a 12 años de estudio (ambos con la misma frecuencia, $28,9 \%)$, casadas $(45,0 \%)$, conviviendo en familia miembro (34,4\%), jubilado (79,8\%). La prevalencia del uso de BZDs fue del 33,6\% ( $n=99$ ). La principal queja de ansiedad y el diagnóstico de trastornos de ansiedad se asociaron con el uso de BZDs.

Conclusiones: la prevalencia del uso de BZDs fue alta y se asoció con el sintoma principal de ansiedad y los diagnósticos de ansiedad y trastornos depresivos, aunque estos se consideran fármacos potencialmente inapropiados y con alto riesgo de complicaciones. El tratamiento de la población geriátrica a menudo se pone en un segundo plano, no recibiendo una atención adecuada dirigida a las demandas específicas que necesitan estos individuos. El conocimiento epidemiológico de la población anciana expone características y ayuda al desarrollo de un plan terapéutico diferenciado.

Palabras clave: anciano, psiquiatría geriátrica, receptores de benzodiazepina, lista de medicamentos potencialmente inapropiados.

\section{Introdução}

A população idosa em países em desenvolvimento, como o Brasil, é composta por individuos com 60 anos ou mais e em países desenvolvidos por aqueles a partir de 65 anos. ${ }^{1}$ No mundo, estima-se que o número de idosos alcance 1,5 bilhões de individuos até 2050, representando um aumento de 9,3\% em 2020 para 16,0\% em 2050. ${ }^{2}$ A população idosa brasileira representa 14,6\%, segundo a Pesquisa Nacional por Amostra de Domicilios Contínua, ${ }^{3}$ o equivalente a 30,3 milhões de pessoas, dado esse que supera a estimativa baseada no Censo de 2010. Os estados que lideram o aumento são o Rio de Janeiro e o Rio Grande do Sul, ambos com proporção de idosos de 18,6\%. ${ }^{4}$

O processo de envelhecimento pode estar associado a um aumento de doenças crônico-degenerativas e, consequentemente, ampliação no número de medicamentos utilizados, podendo incluir, ainda, maior perda de funções, redução da autonomia e mortalidade. ${ }^{5}$ A saúde mental e o bem-estar emocional são tão importantes na velhice como em qualquer outro periodo da vida. Mais de 20\% das pessoas acima dos 60 anos de idade sofrem de transtorno mental, dentre eles os transtornos de ansiedade afetam 3,8\% dos idosos e o uso abusivo de substâncias psicotrópicas em torno de 1\%. ${ }^{6}$

Para o tratamento de ansiedade, insônia e agitação em idoso, a classe dos benzodiazepínicos (BZDs) é usualmente prescrita, o que representa uma preocupação, pois o uso crônico dessa classe de medicamento está relacionado a déficit cognitivo e psicomotor, quedas e risco de fraturas; portanto, sua prescrição é considerada potencialmente inadequada para idosos. ${ }^{7}$ No município de Bambuí (MG, Brasil) foi desenvolvido um estudo com 1.606 participantes e observaram uma frequência de utilização de BZDs em idosos de $22 \%$ (idade média 69 anos). Os BZDs utilizados eram de meia vida longa e o tempo de utilização maior que um ano. ${ }^{8}$ Outro estudo realizado com 550 idosos de 60 a 103 anos, em Porto Alegre (RS, Brasil), demonstrou uma prevalência de utilização de BZDs de 7,3\%. Nessa amostra, 38,2\% dos idosos tinham diagnóstico psiquiátrico, desses $28,8 \%$ de depressão e 20,2\% de ansiedade. O uso de BZDs foi associado com depressão, ansiedade, depressão/ansiedade e transtorno bipolar e, ainda, com uso concomitante de antidepressivos. Os indivíduos que autorrelataram a utilização de álcool apresentaram menor risco de utilizar BZDs. ${ }^{9}$

Os medicamentos potencialmente inapropriados (MPIs) recebem essa denominação por se tratarem de medicamentos cuja evidência de benefício é inferior aos efeitos colaterais nos idosos, 
além de possuir outras alternativas para substituição. ${ }^{5}$ A Sociedade Americana de Geriatria desenvolveu uma lista de MPIs que devem ser evitados nos idosos na maioria das circunstâncias ou em situações específicas, como em certas doenças ou condições. É chamada também de Critério de Beers e enfatiza que o uso de BZDs se enquadra na recomendação de MPIs para idosos, devido ao aumento do risco de déficit cognitivo, delirium, quedas, fraturas e acidentes automobilisticos. ${ }^{5}$

Esse artigo tem como objetivo verificar a prevalência de uso de medicamentos BZDs na população idosa atendida em um ambulatório de psiquiatria geriátrica de um hospital universitário em Porto Alegre, Brasil.

\section{Métodos}

\section{Delineamento}

Estudo transversal, realizado no ambulatório de Psiquiatria Geriátrica do Hospital São Lucas da Pontifícia Universidade do Rio Grande do Sul (PUCRS), localizado em Porto Alegre (RS, Brasil). Esse estudo está inserido no projeto de pesquisa Programa de Envelhecimento e Saúde Mental (PESM), com participação de médicos em formação psiquiátrica, acadêmicos em iniciação cientíica, mestrandos, doutorandos e pós-doutorandos.

\section{Amostra estudada}

Foram incluidos todos os individuos com idade igual ou superior a 60 anos atendidos no ambulatório de geriatria psiquiátrica, no período de março de 2014 a maio de 2019. O ambulatório realiza consultas de pacientes em atendimento particular ou convênios. Um total de 295 participantes foram inseridos no estudo.

O cálculo amostral foi definido em 264 participantes, considerando um tamanho populacional desconhecido, precisão de 5\% e prevalência estimada de utilização de BZDs de $22 \%{ }^{8}$

Como critério de exclusão não foram considerados nesse estudo os indivíduos com dados incompletos.

\section{Coleta de dados}

Os dados foram coletados na primeira consulta psiquiátrica realizada por médicos residentes e cursistas supervisionados por preceptores. Os instrumentos de pesquisa contêm informações sociodemográficas (sexo, idade, escolaridade, estado civil, mora com, aposentadoria, procedência e custeio da consulta), clínicas e de saúde (queixa principal, hipótese diagnóstica, comorbidades clínicas, histórico familiar psiquiátrico e internação psiquiátrica) e uso contínuo de medicamentos (benzodiazepínicos, antidepressivos, antipsicóticos, antiepiléticos, lítio e medicamentos clínicos).

Os diagnósticos psiquiátricos estabelecidos foram realizados com base na entrevista clínica e nos critérios do DSM- $5 .{ }^{10}$ As seguintes hipóteses diagnósticas foram identificadas: transtornos depressivos; transtornos ansiosos; transtornos afetivo bipolar; esquizofrenias; transtornos da personalidade; transtornos mentais e orgânicos das demências; e transtornos mentais e comportamentais do uso de álcool.

\section{Análise estatistica}

Os dados foram analisados através do programa estatístico SPSS, versão 17. As variáveis foram descritas através de frequências, média e desvio padrão. O teste qui-quadrado de Pearson foi empregado para testar a associação entre as variáveis categóricas. As variáveis ordinais foram comparadas pelo teste de tendência linear do qui-quadrado. Os resultados foram considerados significativos quando $\mathrm{P}<0,05$

\section{Aspectos éticos}

O trabalho foi aprovado pela Comissão Científica do Instituto de Geriatria e Gerontologia da PUCRS (SIPESQ: 8322) e pelo Comitê de Ética em Pesquisa da PUCRS (CAAE 89158218.5.0000.5336, número de parecer 2.823.737). Todos os indivíduos incluídos no estudo assinaram o Termo de Consentimento Livre e Esclarecido. Foram respeitados o sigilo e a liberdade do paciente em permanecer no grupo a ser pesquisado. A pesquisa atende às Diretrizes e Normas Regulamentadoras em Pesquisa, conforme a Resolução 466/12 do Conselho Nacional de Saúde. 


\section{Resultados}

Foram avaliados 295 idosos com média de idade de $72,1 \pm 8,3$ anos, sendo na maioria mulheres (76,6\%), com escolaridade de 5 a 8 e 9 a 12 anos de estudo (ambos com a mesma frequência, 28,9\%), casados $(45,0 \%)$, residindo com familiar $(34,4 \%)$, aposentados $(79,8 \%)$ e procedentes de Porto Alegre (63,7\%). Em relação às consultas atendidas, em $53,2 \%$ a origem foi por encaminhamento de outras especialidades e o custeio ocorreu de forma particular em 51,9\% e convênios 48,1\%.

A prevalência de utilização de BZDs foi de 33,6\% (n=99). Na Tabela 1 foi analisado o uso de
BZDs com variáveis sociodemográficas, pode ser observado que os viúvos utilizam mais BZDs e os divorciados menos $(P=0,046)$. Em relação às variáveis clínicas e de saúde, a queixa principal de ansiedade foi significativamente associada ao uso de BZD ( $P=0,004)$, bem como a hipótese diagnóstica de Transtornos Ansiosos ( $\mathrm{P}=0,037)$ (Tabela 2). O uso concomitante de BZDs com outros medicamentos psicofármacos e clínicos não apresentou associação, exceto o lítio onde nenhum dos idosos que o utilizavam fazia também uso de BZDs $(P=0,042)$ (Tabela 3 ).

Tabela 1 - Comparação do uso de BZDs e variáveis sociodemográficas

\begin{tabular}{|c|c|c|c|}
\hline \multirow{2}{*}{ Variáveis } & População & Usa BZDs & \multirow{2}{*}{$\mathbf{P}$} \\
\hline & $n(\%)$ & $\%$ & \\
\hline \multicolumn{4}{|l|}{ Sexo } \\
\hline Feminino & $226(76,6)$ & 34,1 & \multirow{2}{*}{0,736} \\
\hline Masculino & $69(23,4)$ & 31,9 & \\
\hline \multicolumn{4}{|l|}{ Faixa Etária (anos) } \\
\hline $60-69$ & $120(40,7)$ & 27,5 & \multirow{3}{*}{$0,149^{*}$} \\
\hline $70-79$ & $114(38,6)$ & 39.5 & \\
\hline 80 ou mais & $61(20,7)$ & 34,4 & \\
\hline \multicolumn{4}{|l|}{ Escolaridade (anos) } \\
\hline até 4 & $71(24,7)$ & 28,2 & \multirow{4}{*}{$0,433^{*}$} \\
\hline $5-8$ & $83(28,9)$ & 32,5 & \\
\hline $9-12$ & $83(28,9)$ & 36,1 & \\
\hline 13 ou mais & $50(17,4)$ & 42,0 & \\
\hline \multicolumn{4}{|l|}{ Estado Civil } \\
\hline Solteiro & $25(8,9)$ & 28,0 & \multirow{4}{*}{0,046} \\
\hline Casado & $126(45,0)$ & 31,7 & \\
\hline Divorciado & $38(13,6)$ & 18,4 & \\
\hline Viúvo & $91(32,5)$ & 42,9 & \\
\hline \multicolumn{4}{|l|}{ Mora com } \\
\hline Sozinho & $64(22,0)$ & 29,7 & \multirow{4}{*}{0,420} \\
\hline Familiar & $100(34,4)$ & 38,0 & \\
\hline Companheiro & $99(34,0)$ & 30,3 & \\
\hline Cuidador & $28(9,6)$ & 42,9 & \\
\hline \multicolumn{4}{|l|}{ Aposentado } \\
\hline Sim & $229(79,8)$ & 35,4 & \multirow{2}{*}{0,385} \\
\hline Não & $58(20,2)$ & 29,3 & \\
\hline Total & 295 (100) & 33,6 & \\
\hline
\end{tabular}

"Teste de tendência linear do qui-quadrado. 
Tabela 2 - Comparação do uso de BZDs e variáveis clínicas e de saúde

\begin{tabular}{|c|c|c|c|}
\hline \multirow{2}{*}{ Variáveis } & População & Usa BZDs & \multirow{2}{*}{$\mathbf{P}$} \\
\hline & n (\%) & $\%$ & \\
\hline \multicolumn{4}{|l|}{ Queixa Principal } \\
\hline Sintomas Depressivos & $171(58,6)$ & 35,1 & 0,511 \\
\hline Sintomas Ansiosos & $98(33,6)$ & 44,9 & 0,004 \\
\hline Sintomas Psicóticos & $23(7,9)$ & 21,7 & 0,211 \\
\hline Sintomas Maníacos & $6(2,1)$ & 16,7 & 0,376 \\
\hline Transtornos Neurológicos & $34(11,6)$ & 23.5 & 0,188 \\
\hline Uso/Abuso de Drogas ou Medicamentos & $8(2,7)$ & 25,0 & 0,603 \\
\hline Ideação Suicida & $2(0,7)$ & 0 & 0,313 \\
\hline \multicolumn{4}{|l|}{ Hipótese Diagnóstica } \\
\hline Transtornos Depressivos & $126(42,7)$ & 37,3 & 0,240 \\
\hline Transtornos Ansiosos & $85(28,8)$ & 42,4 & 0,042 \\
\hline Transtornos Afetivo Bipolar & $24(8,1)$ & 16,7 & 0,067 \\
\hline Esquizofrenias & $14(4,7)$ & 14,3 & 0,118 \\
\hline Transtornos da Personalidade & $6(2,0)$ & 33.3 & 0,991 \\
\hline $\begin{array}{l}\text { Transtornos Mentais e Orgânicos: } \\
\text { Demências }\end{array}$ & $34(11,5)$ & 20,6 & 0,089 \\
\hline $\begin{array}{l}\text { Transtornos Mentais e Comportamentais: } \\
\text { uso de álcool }\end{array}$ & $6(2,0)$ & 16,7 & 0,376 \\
\hline \multicolumn{4}{|l|}{ Comorbidades Clínicas } \\
\hline Sim & $256(87,7)$ & 35,5 & \multirow{2}{*}{0,114} \\
\hline Não & $36(12,3)$ & 22,2 & \\
\hline \multicolumn{4}{|l|}{ Histórico Familiar Psiquiátrico } \\
\hline Sim & $112(44,6)$ & 37,5 & \multirow{2}{*}{0,396} \\
\hline Não & $139(55,4)$ & 32,4 & \\
\hline \multicolumn{4}{|l|}{ Internação Psiquiátrica } \\
\hline $\operatorname{Sim}$ & $57(19,5)$ & 35,1 & \multirow{2}{*}{0,770} \\
\hline Não & $236(80,5)$ & 33,1 & \\
\hline Total & $295(100)$ & 33,6 & \\
\hline
\end{tabular}


Tabela 3 - Comparação do uso de BZDs com outros medicamentos utilizados concomitantemente

\begin{tabular}{|c|c|c|c|}
\hline \multirow{2}{*}{ Variáveis } & População & Usa BZDs & \multirow{2}{*}{$\mathbf{P}$} \\
\hline & n (\%) & $\%$ & \\
\hline \multicolumn{4}{|c|}{ Antidepressivos } \\
\hline $\operatorname{Sim}$ & $180(61,0)$ & 36,1 & \multirow{2}{*}{0,246} \\
\hline Não & $115(39,0)$ & 29,6 & \\
\hline \multicolumn{4}{|c|}{ Antipsicóticos } \\
\hline Sim & $83(28,1)$ & 26,5 & \multirow{2}{*}{0,108} \\
\hline Não & $212(71,9)$ & 36,3 & \\
\hline \multicolumn{4}{|c|}{ Antiepiléticos } \\
\hline Sim & $21(7,1)$ & 28,6 & \multirow[b]{2}{*}{0,615} \\
\hline Não & $274(92,9)$ & 33,9 & \\
\hline \multicolumn{4}{|l|}{ Lítio } \\
\hline Sim & $8(2,7)$ & 0 & \multirow[b]{2}{*}{0,042} \\
\hline Não & $287(97,3)$ & 34.5 & \\
\hline \multicolumn{4}{|c|}{ Medicamentos Clínicos } \\
\hline Sim & $249(85,6)$ & 35,7 & \multirow{2}{*}{0,131} \\
\hline Não & $42(14,4)$ & 23,8 & \\
\hline Total & 295 (100) & 33,6 & \\
\hline
\end{tabular}

\section{Discussão}

Os idosos em uso de BZDs corresponderam a 33,6\% dos pacientes atendidos no ambulatório de psiquiatria geriátrica. Resultados semelhantes são encontrados em outros estudos, como realizado em Lleida (Espanha), com 161.125 pessoas, durante 14 anos, (2002 a 2015), que apresentou uma prevalência de dispensação de BZDs de 36,1\% para as pessoas com mais de 65 anos. ${ }^{11}$ No Brasil, na cidade de Bambui (MG, Brasil), um estudo de coorte com 882 idosos, nos anos de 1997 e de 2012, demonstrou um aumento na prevalência do uso de BZDs de $24,9 \%$ para $33.9 \%$, respectivamente..$^{12} \mathrm{O}$ crescente uso de BZDs cria um alerta, baseado em que apenas um terço das prescrições de BZDs nessa faixa etária são consideradas adequadas, ou seja, de uso não indevido e não excessivo. ${ }^{13}$ Os idosos são bastante sensiveis aos BZDs, podendo desenvolver efeitos neurocognitivos semelhantes aos do álcool, bem como apresentar risco aumentado de agitação paradoxal. Dessa forma, sempre que possivel, a sua prescrição deve ser evitada, mas quando necessária, em doses baixas e por um curto periodo. ${ }^{14}$

Um estudo realizado em 2008, com idosos de 65 a 80 anos, observou que $6,1 \%$ dos homens e 10,8\% das mulheres usavam BZDs e a grande maioria prescrito por médicos não psiquiatras. ${ }^{15}$ No presente estudo, os idosos avaliados já faziam uso contínuo de BZDs prescritos por médicos de outras especialidades. É importante o correto acompanhamento e o tratamento desses idosos em ambulatório de psiquiatria geriátrica, que é o local mais adequado para atendimento de maneira especializada e personalizada. ${ }^{16}$

As mulheres, geralmente, apresentam taxas de prevalência mais altas em todos os transtornos de ansiedade e transtornos relacionados em comparação com os homens. ${ }^{17.18}$ Airagnes et al. (2016), cita que gênero feminino, múltiplos prescritores, polifarmácia, dor crônica, deficiência física ou 
mobilidade reduzida, deficiência cognitiva, isolamento social e ideação suicida são fatores de risco individuais associados ao uso de BZDs. ${ }^{13} \mathrm{Em}$ contrapartida, nesse estudo o uso de BZDs em mulheres e homens foi praticamente equivalente. Uma possivel explicação se encontra no fato da amostra avaliada não ser homogênea, ou seja, o número de participantes homens ser menor. Historicamente, os homens costumam procurar menos por serviços de saúde ou somente quando já se apresentam sintomáticos buscam por ajuda. Outro ponto importante a ser avaliado é que a coleta de dados foi realizada em um ambulatório especializado e que recebe pacientes que já iniciaram um tratamento médico.

$\mathrm{Na}$ amostra estudada foi observado que o uso de BZDs por viúvos é maior, o que pode estar relacionado ao processo de luto, sendo que a prescrição de BZDs pode ser realizada para alívio desses sintomas, apesar de existir o alerta de inibir o processo natural. Um estudo no Reino Unido descreveu um risco 9,3\% maior de prescrição de BZDs no ano seguinte ao luto. ${ }^{19}$ Já a população divorciada foi a que menos utilizou BZDs. Estudos anteriores que examinaram o estado civil obtiveram resultados semelhantes. 9.20 Como também, outro trabalho demonstrou uma diminuição significativa dos sintomas ansiosos em idosos divorciados. ${ }^{21} \mathrm{~A}$ resolução de questões como descontentamento com a situação do parceiro e a baixa qualidade da relação conjugal podem estar associadas a um menor risco de transtornos de ansiedade e, consequentemente, de utilização de BZDs.

A maioria dos pacientes em uso de BZDs relataram como queixa principal a ansiedade. Após avaliação psiquiátrica, os transtornos ansiosos, seguidos por transtornos depressivos foram as hipóteses diagnósticas mais associadas com o uso de BZDs. A ansiedade é frequentemente relacionada ao uso de substâncias e transtornos do humor. Os transtornos de ansiedade são frequentemente observados em idosos, mas em grande parte não são detectados e tratados corretamente. Estudos epidemiológicos indicam prevalência que varia de 1,2 a 15\% em individuos que vivem em comunidade e $1 \%$ a $28 \%$ no ce- nário clínico. ${ }^{22}$ Resultados semelhantes foram descritos na Espanha, onde os diagnósticos mais frequentemente associados ao uso de BZDs foram ansiedade (24\%) e depressão (19\%). ${ }^{11}$ Há indicação, mesmo que com restrições, do uso de BZDs para o tratamento da ansiedade e, muitas vezes, essa indicação coexiste no tratamento para ansiedade e depressão concomitantes, provavelmente, por isso eles sejam tão utilizados, mesmo não sendo a primeira linha. ${ }^{23} \mathrm{Em}$ idosos, os BZDs devem ser evitados, sendo indicado o prescritor utilizar outras opções terapêuticas para o tratamento da ansiedade. Os inibidores seletivos da recaptação de serotonina (ISRS) são a primeira linha de tratamento para transtornos ansiosos. ${ }^{23}$ É importante reiteradamente lembrar que a escolha de um medicamento para o tratamento de idosos deve sempre seguir a regra do polegar - comece devagar, vá devagar e titule geralmente até metade da dose adulta. ${ }^{14}$

Estima-se que 52\% dos pacientes com transtorno bipolar têm ansiedade comórbida ou transtorno relacionado. ${ }^{23}$ Nesse estudo, pacientes que faziam uso do modulador de humor lítio não utilizavam concomitantemente BZDs, como também os pacientes com diagnóstico de transtorno de humor bipolar e esquizofrenia foram os que menos utilizaram BZDs. O lítio é um estabilizador de humor e primeira linha para o tratamento de transtorno de humor bipolar, possui um moderado efeito antidepressivo e neuroprotetor. ${ }^{23}$ Existem poucos dados na literatura, sobre o benefício do uso de BZDs no tratamento de transtorno de humor bipolar. Os idosos com esse transtorno e em uso de lítio são mais frequentemente direcionados para tratamento com especialista, por serem considerados quadros mais complexos, onde os médicos de outras especialidades costumam referenciar o atendimento. Um acompanhamento especializado pode explicar o não uso de BZDs por esse grupo.

O uso de MPIs tem sido associado ao aumento do risco de quedas, hospitalizações, comprometimento cognitivo e morte. ${ }^{5}$ Os BZDs são os MPIs mais conhecidos e estudados, mas ainda muito prescritos. ${ }^{24}$ Esse cenário atingiu proporções pandêmicas há décadas e é um importante problema de saúde 
pública, pois o uso crônico desses medicamentos resulta em um aumento nos fatores relacionados com morbidade, risco de quedas, intoxicação e agravamento dos sintomas depressivos e cognitivos. ${ }^{25} \mathrm{~A}$ desprescrição dos BZDs deve ser considerada para todos os idosos. A utilização desse grupo de medicamentos deve ser de curto prazo, porque quando excedem 4 a 6 semanas, podem ocasionar também tolerância, dependência e crises de abstinência, ${ }^{26}$ além dos outros efeitos acima citados.

O estudo apresenta limitações, como a coleta ser realizada durante consulta médica e as respostas dos participantes dependerem do autorrelato. Também, o delineamento do estudo transversal impossibilita estabelecer a temporalidade dos fatores associados.

\section{Conclusão}

Existe uma alta prevalência de uso de BZDs por idosos com queixa de ansiedade e diagnósticos de transtornos ansiosos e depressivos, mesmo essa classe sendo considerada MPIs para a população idosa. O tratamento de idosos muitas vezes não é prioritário, não recebendo devidamente os cuidados direcionados às demandas que tal população exige e gerando risco. O conhecimento epidemiológico da população idosa expõe características e auxilia na elaboração de estratégias de atuação voltada ao grupo, visando a qualidade do tratamento e de vida.

O interesse em desbravar a psiquiatria geriátrica é factual em estudos nacionais e internacionais, outrossim, novas evidências sobre efeitos de medicamentos largamente utilizados sem segurança estabelecida e crescente número de diagnósticos psiquiátricos nesta faixa etária. Por conseguinte, a otimização de tratamentos pode ser obtida com o conhecimento, por médicos e demais profissionais de saúde, para auxiliar nos tratamentos prestados, evitando iatrogenias.

\section{Agradecimentos}

O presente trabalho foi realizado com apoio da Coordenação de Aperfeiçoamento de Pessoal Nivel Superior - Brasil (CAPES) - Código de Financiamento 001.

\section{Fonte de financiamento}

O presente trabalho foi realizado com apoio da Coordenação de Aperfeiçoamento de Pessoal Nivel Superior - Brasil (CAPES) - Código de Financiamento 001

\section{Conflitos de interesse}

Os autores declaram não haver conflitos de interesses ao conteúdo deste estudo.

\section{Contribuição dos autores}

Isabella Serafin Couto: escrita, organização do banco de dados, análise estatística, discussão dos resultados e aprovação da versão final.

Vanessa Sgnaolin: escrita, coleta de dados, organização do banco de dados, análise estatística, discussão dos resultados e aprovação da versão final.

Paula Engroff: escrita, coleta de dados, organização do banco de dados, análise estatistica, discussão dos resultados e aprovação da versão final.

Letícia Güenter Dannebrock: coleta de dados, organização do banco de dados, discussão dos resultados, revisão e aprovação da versão final.

Luiz Gustavo Guilhermano: discussão dos resultados, revisão e aprovação da versão final.

Alfredo Cataldo Neto: coordenação do projeto de pesquisa, discussão dos resultados e aprovação da versão final.

\section{Referências}

1. Active ageing: A policy framework [Internet]. Genebra: World Health Organization. 2002 [cited 2020 Out 25]. Available from: https://apps.who.int/iris/handle/10665/67215.

2. World Population Prospects [Internet]. New York: United Nations. 2019 [cited 2020 Out 25]. Available from https://population.un.org/wpp.

3. Sintese de indicadores sociais: uma análise das condições de vida da população brasileira, 2017 [Internet]. Rio de Janeiro: Instituto Brasileiro de Geografia e Estatística. 2017 [cited 2020 Out 15]. Available from: https:// biblioteca.ibge.gov.br/visualizacao/livros/liv101459.pdf.

4. Perissé C, Marli M. Caminhos para uma melhor idade. Retratos a revista do IBGE. 2019, 16:18-27.

5. By the 2019 American Geriatrics Society Beers Criteria $\AA$ Update Expert Panel. American Geriatrics Society 2019 Updated AGS Beers Criteria ${ }^{\circledR}$ for Potentially Inappropriate Medication Use in Older Adults. J Am Geriatr Soc. 2019;67(4):674-94. 
6. Organização Mundial da Saúde. La salud mental y los adultos mayores. Genebra: World Health Organization; 2017.

7. laboni A, et al. Changing Pattern of Sedative Use in Older Adults: A Population-Based Cohort Study. Drugs \& aging. 2016:33(7):523-33

8. Alvarenga JM, et al. Prevalence and sociodemographic characteristics associated with benzodiazepines use among community dwelling older adults: the Bambui Health and Aging Study (BHAS). Revista Brasileira de Psiquiatri. 2008;30(1):7-11.

9. Sgnaolin V, et al. Patterns of chronic benzodiazepine use in the elderly. Rev Psiquiatr Clín. 2016;43(4):79-82.

10. American Psychiatric Association. DSM-5: Manual diagnóstico e estatístico de transtornos mentais. Porto Alegre: Artmed Editora; 2014

11. Torres-bondia F, et al. Trends in the consumption rates of benzodiazepines and benzodiazepine-related drugs in the health region of Lleida from 2002 to 2015. BMC Public Health. 2020;20(1):818.

12. Oliveira ALML. Estudo de tendência do uso de benzodiazepínicos entre idosos residentes no município de Bambuí, Minas Gerais [dissertação]: Programa de Pós-Graduação em Ciências da Saúde, Instituto René Rachou, Fundação Oswaldo Cruz; 2018. 61 f. Disponivel em: https://www.arca.fiocruz.br/handle/icict/34254.

13. Airagnes G, et al. Benzodiazepine Misuse in the Elderly: Risk Factors, Consequences, and Management. Curr Psychiatry Rep. 2016;18(10):89.

14. Subramanyam A A, et al. Clinical practice guidelines for Geriatric Anxiety Disorders. Indian Journal of Psychiatry. 2018; 60(suppl. 3):S371-S382.

15. Olfson M, King M, Schoenbaum M. Benzodiazepine Use in the United States. JAMA Psychiatry. 2015:72(2):136.

16. Borja-oliveira C R, Assato C P. Psicofármacos potencialmente inapropriados para idosos. Estud Interdiscipl Envelhec. 2015:20(3):687-701.

17. Costa COD, et al. Prevalência de ansiedade e fatores associados em adultos. J Bras Psiquiatr. 2019;68(2):92-100.

18. Kinrys G, Wygant L. E. Anxiety disorders in women: does gender matter to treatment? Braz J Psychiatry. 2005: 27(suppl 2):S43-50.

19. Cook JM, Biyanova T., Marshall R. Medicating Grief With Benzodiazepines: Physician and Patient Perspectives. Arch Intern Med. 2007:167(18):2006-7.

20. Manthey L, et al. Correlates of (inappropriate) benzodiazepine use: the Netherlands Study of Depression and Anxiety (NESDA). Br J Clin Pharmacol. 2011;71(2):263-72.

21. Boehlen FH, et al. Gender-specific predictors of generalized anxiety disorder symptoms in older adults: Results of a large population-based study. J Affect Disord Rep. 2020; 262:174-81.
22. Nardi TC, Grassi-Oliveira R., Pádua AC. Terapia Cognitivo-Comportamental no Tratamento dos Sintomas de Ansiedade em Idosos. In: Freitas ER, Barbosa AJG, Neufeld CB, org. Terapias cognitivo-comportamentais com idosos. Novo Hamburgo: Sinopsys; 2016.

23. Kennedy SH, et al:; CANMAT Depression Work Group. Clinical guidelines for the treatment of depressive disorders. Can J Psychiatry. 2001;46(suppl 1):5S-90S.

24. Carrasco-Garrido P, et al. Psychotropics use in the Spanish elderly: predictors and evolution between years 1993 and 2003. Pharmacoepidemiol Drug Saf. 2007:16(4):449-57.

25. Richardson K, Bennett K, Kenny RA. Polypharmacy including falls risk increasing medications and subsequent falls in community-dwelling middle-aged and older adults. Age Ageing. 2015;44:90-6.

26. Bloomfield HE, et al. Deprescribing for Community-Dwelling Older Adults: a Systematic Review and Meta-analysis. J Gen Intern Med. 2020;35(11):3323-32.

\section{Isabella Serafin Couto}

Médica pela Universidade do Extremo Sul Catarinense (UNESC), em Criciúma, SC, Brasil; especialista em Psiquiatria pela Pontifícia Universidade Católica do Rio Grande do Sul (PUCRS), em Porto Alegre, RS, Brasil.

\section{Vanessa Sgnaolin}

Doutora em Gerontologia Biomédica pela Pontificia Universidade Católica do Rio Grande do Sul (PUCRS), em Porto Alegre, RS, Brasil; mestre em Medicina e Ciências da Saúde pela Pontificia Universidade Católica do Rio Grande do Sul (PUCRS), em Porto Alegre, RS, Brasil.

\section{Paula Engroff}

Doutora em Gerontologia Biomédica pela Pontificia Universidade Católica do Rio Grande do Sul (PUCRS), em Porto Alegre, RS, Brasil.

\section{Letícia Güenter Dannebrock}

Médica pela Universidade Luterana do Brasil (ULBRA), em Canoas, RS, Brasil; especialista em Psiquiatria pela Pontifícia Universidade Católica do Rio Grande do Sul (PUCRS), em Porto Alegre, RS, Brasil.

\section{Luiz Gustavo Guilhermano}

Mestre em Farmacologia pela Universidade Federal de Ciências da Saúde de Porto Alegre (UFCSPA), em Porto Alegre, RS; Psiquiatra Titular da Associação Brasileira de Psiquiatria (ABP), em Rio de Janeiro, RJ; professor da Pontifícia Universidade Católica do Rio Grande do Sul (PUCRS), em Porto Alegre, RS, Brasil. 


\section{Alfredo Cataldo Neto}

Doutor em Medicina e Ciências da Saúde pela Pontificia Universidade Católica Do Rio Grande do Sul (PUCRS), em Porto Alegre, RS, Brasil; professor da Pontifícia Universidade Católica do Rio Grande do Sul (PUCRS), em Porto Alegre, RS, Brasil.

\section{Endereço para correspondência}

\section{Vanessa Sgnaolin}

Pontificia Universidade Católica do Rio Grande do Sul

Av. Ipiranga, 6681, prédio 40, $8^{\circ}$ andar, sala 804

90619-900

Porto Alegre, RS, Brasil

Os textos deste artigo foram revisados pela Poá Comunicação e submetidos para validação do(s) autor(es) antes da publicação. 